ARTÍCULO ORIGINAL

\title{
SITUACIÓN DE LA COMORBILIDAD TUBERCULOSIS Y DIABETES EN PERSONAS ADULTAS EN EL PERÚ, 2016-2018
}

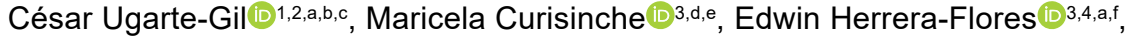 \\ Henry Hernandez $\mathbb{1}^{3, a}$, Julia Rios $\mathbb{1}^{3, a}$ \\ 1 Instituto de Medicina Tropical Alexander von Humboldt, Facultad de Medicina, Universidad Peruana Cayetano Heredia, \\ Lima, Perú. \\ 2 TB Centre, London School of Hygiene and Tropical Medicine, Londres, Reino Unido. \\ 3 Centro Nacional de Salud Pública, Instituto Nacional de Salud, Lima, Perú \\ ${ }^{4}$ Servicio de Neumología, Hospital Nacional Arzobispo Loayza, Lima, Perú. \\ a Médico cirujano; ${ }^{\text {b }}$ magíster en Epidemiología; ${ }^{\text {c }}$ doctor en Epidemiología y Control de Enfermedades Globales; \\ d licenciada en Enfermería; ${ }^{\mathrm{e}}$ magíster en Ciencias-Salud Pública; ${ }^{\mathrm{f}}$ médico neumólogo.
}

\section{RESUMEN}

Objetivo: Describir las características de los pacientes adultos con tuberculosis (TB) y diabetes mellitus (DM) en el Perú, y explorar la asociación de la DM y la mortalidad en personas con TB. Materiales y métodos: Se realizó un análisis secundario de la base de datos del Sistema de Información Gerencial de Tuberculosis de la Dirección de Prevención y Control de Tuberculosis del Ministerio de Salud de Perú. Se incluyeron aquellos pacientes adultos que iniciaron tratamiento con el esquema de TB sensible a los medicamentos los años 2016, 2017 y 2018. Se realizó un análisis descriptivo de los pacientes con TB con DM y un análisis exploratorio para evaluar la asociación de la DM con la mortalidad usando una regresión de Poisson para determinar el riesgo relativo (RR). Resultados: Se registraron 67524 adultos con TB sensible a los medicamentos, de los cuales se reportaron $6529(9,7 \%)$ personas como TB con DM y $4048(6,0 \%)$ con infección por VIH. De los pacientes reportados con TB con DM, la mayoría eran hombres $(60,2 \%)$ con una mediana de edad de 53 años. Con relación a la mortalidad, las personas con TB con DM tuvieron una mayor frecuencia de muerte comparado con aquellas personas con TB sin DM (7,2\% vs. $5,4 \%)$. En el análisis exploratorio de factores asociados a la mortalidad, la DM presentó un RR crudo de 1,32 (IC 95\%: 1,20-1,50); sin embargo, esta asociación varió en el modelo ajustado con un RR de 0,93 (IC 95\%: 0,84-1,04). Conclusiones: La DM es la comorbilidad más frecuente en pacientes con TB en el Perú, aunque no se encontró asociación con una mayor mortalidad.

Palabras clave: Tuberculosis; Diabetes Mellitus; Comorbilidad; Mortalidad; Sobrevida (fuente: DeCS BIREME).

\section{SITUATION OF THE TUBERCULOSIS-DIABETES COMORBIDITY IN ADULTS IN PERU: 2016-2018}

\begin{abstract}
Citar como: Ugarte-Gil C, Curisinche M, Herrera-Flores E, Hernandez $\mathrm{H}$, Rios J. Situación de la comorbilidad tuberculosis y diabetes en personas adultas en el Perú, 2016-2018. Rev Peru Med Exp Salud Publica. 2020;38(2):25460. doi: https://doi.org/10.17843/ rpmesp.2021.382.6764.
\end{abstract}

Correspondencia: César UgarteGil; Instituto de Medicina Tropical Alexander von Humboldt, Universidad Peruana Cayetano Heredia, Av. Honorio Delgado 430, Lima, Perú; cesar.ugarte@upch.pe

Recibido: $17 / 11 / 2020$ Aprobado: 02/06/2021 En línea: 08/07/2021

\section{ABSTRACT}

Objective: To describe the characteristics of adult patients with tuberculosis (TB) and diabetes mellitus (DM) in Peru, and to explore the association of DM and mortality in people with TB. Materials and methods: We carried out a secondary analysis of the database of the Management Information System of Tuberculosis of the Tuberculosis Prevention and Control Directorate of the Ministry of Health of Peru. Adult patients who started treatment with the scheme for drug-sensitive TB in 2016, 2017 and 2018 were included. We carried out a descriptive analysis of patients with TB and DM, and an exploratory analysis to assess the association of DM with mortality using a Poisson regression to determine the relative risk (RR). Results: We registered 67,524 adults with drug-sensitive TB, of which 6,529 (9.7\%) people were reported as having TB and DM; and 4,048 (6.0\%) had HIV infection. Of the patients reported with TB and DM, most were men $(60.2 \%)$ with a median age of 53 years. Regarding mortality, people with TB and DM had a higher frequency of death compared to those with TB without DM (7.2\% vs 5.4\%). In the exploratory analysis of factors associated with mortality, DM had a crude RR of 1.32 (95\% CI: 1.20-1.50); however, this association varied in the adjusted model with a RR of 0.93 (95\% CI: 0.84-1.04). Conclusions: DM is the most frequent comorbidity in patients with TB in Peru, although no association with higher mortality was found.

Keywords: Tuberculosis; Diabetes Mellitus; Comorbidity; Mortality; Survival (source: MeSH NLM). 


\section{INTRODUCCIÓN}

En los últimos años se está evidenciando un aumento de las enfermedades no transmisibles como la diabetes (DM) en poblaciones de países con ingresos medios y bajos como el Perú ${ }^{(1)}$. El hecho de que el Perú sea uno de los países con más carga de tuberculosis (TB) en las Américas, lo pone en una situación de alerta ante un posible aumento de casos de comorbilidad TB con $\mathrm{DM}{ }^{(2)}$. Una revisión sistemática de 200 estudios encontró una prevalencia combinada de DM en pacientes con TB del 15,3\%, donde el estimado para América Latina fue del $7,7 \%{ }^{(3)}$.

Esta comorbilidad puede poner en riesgo los esfuerzos que se vienen realizando para controlar la TB. Un modelo matemático determinó cómo el aumento de la prevalencia de DM podría poner en peligro la reducción de la incidencia de TB observada durante los últimos años. Con una prevalencia general de DM del 10\% (según las estimaciones de la Federación Internacional de Diabetes en 2013), la incidencia de TB aumentaría en un 3\% en el 2035, en comparación con la incidencia estimada sin un aumento en la prevalencia de $\mathrm{DM}^{(4)}$. Asimismo, si se considera que la DM es una enfermedad multifactorial y que los factores, como la obesidad, se están incrementando, el escenario de la prevalencia de DM sería del $12,5 \%$, con un cambio en la incidencia de TB del $8 \%$ en el 2035. Este modelo demuestra que el aumento de la prevalencia de DM puede afectar directamente la incidencia de $\mathrm{TB}$ en los próximos años, lo cual repercutiría negativamente en las metas establecidas para eliminar la $\mathrm{TB}^{(4)}$.

Existe evidencia que la comorbilidad TB con DM aumenta el riesgo de malos resultados en el tratamiento de TB. Una revisión sistémica reciente encontró que la DM aumenta el riesgo de muerte (OR: 1,88; IC 95\%: 1,59-2,21) y de recaídas (OR: 1,64; IC 95\%: 1,29-2,08) ${ }^{(5)}$. Otra revisión sistemática encontró que los pacientes con DM tienen entre dos y cuatro veces más riesgo de tener TB activa ${ }^{(6)}$. Asimismo, en el Reino Unido se ha encontrado que el tener antecedentes de TB aumenta en casi seis veces el riesgo de DM, por lo que esta comorbilidad potencia el impacto clínico en el paciente aun después de tener el episodio de $\mathrm{TB}^{(7)}$. Finalmente, la presencia de DM en pacientes con TB incrementa el riesgo de muerte cardiovascular, cinco años después de haber terminado el tratamiento de TB (HR: 1,70; IC 95\%: 1,23-2,35) ${ }^{(8)}$.

Parte de estos resultados se explican por el hecho de que la DM, por la hiperglicemia, crea cambios inflamatorios y vasculares que pueden incrementar el riesgo de TB, además de cambios en la respuesta inmune ${ }^{(9)}$. Asimismo, existen estudios que muestran evidencia que el control de la glicemia puede disminuir dicho riesgo ${ }^{(10)}$, siendo esta evidencia importante para el tratamiento de la comorbilidad de TB con DM.

En este contexto es importante evaluar las características epidemiológicas de los pacientes con TB y DM en el Perú, con el objetivo de tener un mejor panorama para la

\section{MENSAJES CLAVE}

Motivación para realizar el estudio: La prevalencia de diabetes mellitus (DM) está en aumento en todo el mundo, especialmente en países como el Perú; lo que puede afectar el control de la tuberculosis (TB).

Principales hallazgos: La DM es la comorbilidad más frecuente en los pacientes con TB, con una mediana de edad mayor en comparación con los pacientes con TB sin DM. A pesar de una mayor frecuencia de mortalidad en pacientes con TB con DM, no se ha encontrado evidencia de asociación de la $\mathrm{DM}$ con la mortalidad de pacientes con TB.

Implicancias: Es frecuente la comorbilidad de TB con DM en pacientes peruanos, se deben evaluar intervenciones que ayuden al manejo de esta comorbilidad.

planificación de potenciales intervenciones para su manejo integral. Este estudio tiene como objetivo describir las características demográficas y del tratamiento de la TB en población adulta con la comorbilidad TB y DM, además de explorar la asociación entre DM y mortalidad en pacientes con TB.

\section{MATERIALES Y MÉTODOS}

\section{Diseño del estudio}

Este es un estudio observacional tipo cohorte retrospectiva basado en un análisis secundario de la base de datos del Sistema de Información Gerencial de Tuberculosis (SIGTB), del Perú.

\section{Fuente de datos}

La base de datos del SIGTB ${ }^{(11)}$ de la Dirección de Prevención y Control de Tuberculosis (DPCTB) del Ministerio de Salud (MINSA), registra a los pacientes de todo el país con tratamiento contra la TB. En esta base de datos se incluyen a pacientes del MINSA, Seguro Social (EsSalud), Sanidad de Fuerzas Armadas y Policiales y del Instituto Nacional Penitenciario (INPE). Previa solicitud, la DPCTB brindó esta base de datos, en archivo de Excel y sin identificadores personales para el análisis de este estudio.

\section{Criterios de selección}

Se incluyeron aquellos pacientes que iniciaron tratamiento los años 2016, 2017 y 2018; mayores de 18 años e incluidos en el esquema de tratamiento para TB sensible a los medicamentos. No se excluyó ningún paciente que cumpliera los criterios de inclusión. 


\section{Variables}

Se consideró como caso de TB con DM a aquellos que tuvieran registro de diagnóstico previo de $\mathrm{DM}$ y/o diagnóstico de DM con prueba de glicemia según el reporte del SIGTB, el cual es incluido en la historia clínica del paciente con TB. Según la Norma Técnica de la DPCTB, todo paciente con diagnóstico de TB (con antecedente de DM, o no) debe realizarse una prueba de glucosa en ayunas, además de otras pruebas de laboratorio como hemograma completo, perfil hepático o prueba para VIH ${ }^{(12)}$.

\section{Análisis estadístico}

Se utilizaron frecuencias para describir variables categóricas (sexo, grupo etario, localización de la enfermedad, institución que brinda la atención, zona geográfica, resultado de tratamiento) así como medianas y rango intercuartil para variables continuas (edad). En el caso del resultado al tratamiento se usaron las categorías indicadas en la Norma Técnica (curado, tratamiento completo, muerte, falla al tratamiento, pérdida en el tratamiento), las cuales concuerdan con las definiciones de resultado al tratamiento de TB sugerido por la Organización Mundial de la Salud (OMS) ${ }^{(12,13)}$. Otras variables evaluadas fueron infección por VIH, antecedente de tratamiento de TB, reacción adversa a medicamentos y reporte de tabaquismo, alcoholismo y drogadicción según la definición que se usa para el llenado del SIGTB basado en lo solicitado por la Norma Técnica de la DPCTB ${ }^{(12)}$.

Para evaluar las diferencias entre los pacientes con $\mathrm{TB}$ sin DM y TB con DM, se utilizó la prueba de Chi cuadrado (variables categóricas) y la prueba de Mann-Whitney (variables numéricas). Se realizó un análisis exploratorio para evaluar factores asociados a la mortalidad, usando una regresión de Poisson con varianza robusta para determinar el riesgo relativo (RR) con intervalos de confianza al 95\% (IC 95\%), y se evaluaron un modelo crudo y uno ajustado por VIH, sexo, grupo etario, localización de TB y antecedente de TB. Se seleccionaron estas variables porque se las reportó como variables de confusión, según la evidencia epidemiológica disponible ${ }^{(14-17)}$. El análisis se realizó usando el paquete estadístico STATA $16.1^{\circ}$ (StataCorp, EUA). Debido a que se analizó una base operacional de todo el país con control de calidad, varias de las variables presentan datos faltantes, aunque menor al 10\%; sin embargo, la información de DM tuvo un $14,5 \%$ de datos faltantes, pero no de forma aleatoria (había regiones que tenían diferente proporción de llenado), así que no se pudo realizar imputación múltiple y solo se ha descrito la información.

\section{Criterios éticos}

Este estudio tuvo la autorización del Comité Institucional de Ética de la Universidad Peruana Cayetano Heredia (SIDISI 200531) y está registrado en PRISA del Instituto Nacional de Salud (registro EI00001745).

\section{RESULTADOS}

Se registraron 90468 pacientes con TB en el SIGTB (Figura 1), de estos 81807 eran adultos. Se excluyeron aquellos que no estuvieran en el esquema de tratamiento para TB sensible a los medicamentos y que no tuvieran resultado favorable al tratamiento. Quedaron 67524 personas con TB, cuyas características sociodemográficas se observan en la Tabla 1 . Se encontró que el 9,7\% de los pacientes tenían DM (6529/67 524) y el 6,0\% de los pacientes tenían VIH (4048/67 524). La descripción de los pacientes con TB con DM se presenta en la Tabla 2.

Las regiones que tenían mayor proporción de pacientes con TB y DM fueron Tumbes, Moquegua, Piura, Ucayali, San Martin y Cajamarca. Con relación a las instituciones, se encontró una frecuencia de TB con DM en EsSalud del 13,1\% (1570/12 018), en la Sanidad de la Policía Nacional del Perú del $12,4 \%(10 / 81)$ y del $9,8 \%$ en el MINSA (4860/49 430) dentro de su población con TB. El INPE informó 1,4\% de $\mathrm{TB}$ con DM, pero presentaba $66,8 \%$ de su población con TB sin resultado y/o reporte de DM (3929/5879). La Sanidad de la Fuerza Aérea (1 paciente) y la Sanidad del Ejercito (14 pacientes) no reportaron pacientes con $\mathrm{TB}$ con $\mathrm{DM}$.

Con relación a los resultados al tratamiento para $\mathrm{TB}$, se encontró que los pacientes con TB con DM tuvieron similar proporción de resultado exitoso (definido como curado y tratamiento terminado): $86,8 \%$ de las personas con TB y DM concluyeron con éxito el tratamiento, comparado con personas con TB sin DM (86,2\%); con relación a la mortali-

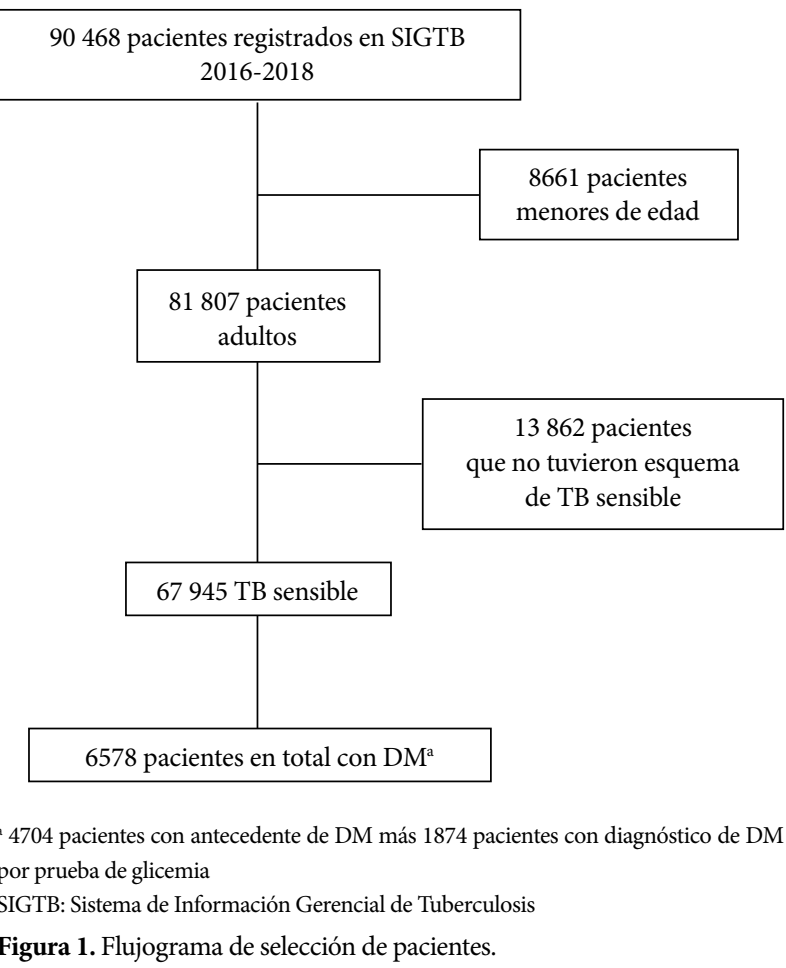


Tabla 1. Características generales de la población con tuberculosis sensible a los medicamentos en el Perú, 2016-2018.

\begin{tabular}{|c|c|}
\hline Características & $\begin{array}{c}\text { Personas con } \mathrm{TB} \\
\quad(\mathrm{n}=67524)\end{array}$ \\
\hline Hombres (\%) & $43486(64,4)$ \\
\hline Mediana de la edad (RIC) & $35(25-52)$ \\
\hline \multicolumn{2}{|l|}{ Grupo etarios $(n=67355)(\%)$} \\
\hline $18-24$ & $16476(24,5)$ \\
\hline $25-34$ & $17128(25,4)$ \\
\hline $35-44$ & $10680(15,9)$ \\
\hline $45-54$ & $7774(11,6)$ \\
\hline $55-64$ & $6297(9,4)$ \\
\hline$\geq 65$ & $9000(13,4)$ \\
\hline Sin antecedente de TB (\%) & $59280(87,8)$ \\
\hline Infección por VIH $(\mathrm{n}=62988)(\%)$ & $4048(6,0)$ \\
\hline Diabetes $(\mathrm{n}=57$ 736) $(\%)$ & $6529(9,7)$ \\
\hline \multicolumn{2}{|l|}{ Institución (\%) } \\
\hline MINSA & $49430(73,2)$ \\
\hline EsSalud & $12018(17,8)$ \\
\hline INPE & $5879(8,7)$ \\
\hline Fuerzas Armadas y Policiales & $197(0,3)$ \\
\hline \multicolumn{2}{|l|}{ Localización de TB (\%) } \\
\hline Pulmonar & $53893(79,8)$ \\
\hline Extrapulmonar & $13631(20,2)$ \\
\hline Tabaquismo & $6218(9,2)$ \\
\hline Alcoholismo & $7810(11,6)$ \\
\hline Drogadicción & $6916(10,2)$ \\
\hline Irregularidad en $1 .{ }^{a}$ fase $(\%)$ & $3652(5,4)$ \\
\hline Irregularidad en $2 .{ }^{a}$ fase (\%) & $9132(13,5)$ \\
\hline \multicolumn{2}{|l|}{ Condición de egreso (\%) } \\
\hline Curado & $44196(65,5)$ \\
\hline Tratamiento completo & $13626(20,2)$ \\
\hline Perdido en el tratamiento & $5670(8,4)$ \\
\hline Fallecido & $3831(5,7)$ \\
\hline Fracaso al tratamiento & $201(0,3)$ \\
\hline Reacción adversa a medicamentos & $716(1,1)$ \\
\hline
\end{tabular}

RIC: rango intercuartil; TB: tuberculosis; VIH: virus de inmunodeficiencia humana; MINSA: Ministerio de Salud; EsSalud: Seguro Social de Salud; INPE: Instituto Nacional Penitenciario

dad durante el tratamiento de TB, los pacientes TB con DM tienen una mayor frecuencia de muerte comparado con $\mathrm{TB}$ $\sin \mathrm{DM}(7,1 \%$ vs. $5,4 \%)$.

En el análisis exploratorio de los factores asociados a la mortalidad, la DM presenta, en el análisis crudo, un RR de 1,32 (IC 95\%: 1,20-1,50); sin embargo, esta asociación varió al ajustarlo por VIH, sexo, grupo etario, tuberculosis extrapulmonar y antecedente de TB (RR: 0,93; IC 95\%: 0,841,04) (Tabla 3).

\section{DISCUSIÓN}

Se encontró que durante el periodo 2016-2018 los peruanos adultos que tuvieron $\mathrm{TB}$ sensible presentaron una mayor prevalencia de DM comparado con $\mathrm{VIH}$, e incluso comparado con adicciones como el alcoholismo, tabaquismo y drogadicción, colocándola como la comorbilidad más común entre las registradas en el SIGTB. Sin embargo, a pesar de que se encontró una mayor frecuencia de mortalidad en pacientes con TB y DM, dicha mortalidad no estaba asociada al estado de la DM. Este resultado es diferente a lo encontrado en otros estudios ${ }^{(5,18)}$, lo que se explicaría por la presencia de variables de confusión no medidas (tratamiento para DM, índice de masa corporal al diagnóstico de TB) que hubieran permitido aislar mejor el efecto de la DM en la mortalidad de pacientes con TB.

Estos resultados sobre la frecuencia de DM son similares a las encontradas en estudios peruanos que evaluaron la asociación de TB y DM, donde se han encontrado prevalencias del $12,5 \%{ }^{(19)}$ y el $14,0 \%{ }^{(20)}$, respectivamente; sin embargo, en estos estudios se realizaron exámenes de hemoglobina glicosilada para confirmar el estado de la DM. Es probable que en el presente estudio exista un subregistro de los casos con DM debido a que no se usa la hemoglobina glicosilada para el diagnóstico. Estos hallazgos de alta prevalencia de DM en pacientes con $\mathrm{TB}$ también se han encontrado en otros países ${ }^{(21,22)}$ donde se han descrito prevalencias de alrededor del $10 \%$.

Se ha reportado que en la mayoría de estos estudios una de las limitaciones más importantes es la falta de datos sobre las comorbilidades no infecciosas (hipertensión arterial, obesidad, depresión, enfermedad cardiovascular), que suelen estar asociadas con DM y a un mayor grado de severidad de esta ${ }^{(23)}$. Dentro del enfoque centrado en la persona, se debe reconocer que la persona que tiene TB debe evaluarse de forma integral, especialmente si se identifican factores de riesgo cardiovasculares. Existe evidencia que incluso luego del tratamiento de $\mathrm{TB}$, existe un incremento en el riesgo en mortalidad. Un estudio en Brasil encontró que el tener DM estuvo asociado con mortalidad por causas cardiovasculares luego de cinco años del diagnóstico de TB (HR: 1,70; IC 95\%: 1,23-2,34) ${ }^{(8)}$. Asimismo, se debe considerar que además de la DM, hay otras condiciones cardiovasculares que afectan a las personas con TB. Se ha encontrado evidencia de la asociación entre la infección por Mycobacterium tuberculosis y el infarto de miocardio ${ }^{(24)}$, por lo que en un contexto donde nuestras poblaciones se enfrentan al aumento de prevalencia en enfermedades no comunicables y la prevalencia aun alta de $\mathrm{TB}$, se debe evaluar de forma integral al paciente.

Como se mencionó, la falta asociación entre la DM y la mortalidad puede deberse a que existen variables de confusión no medidas. Un ejemplo es el tratamiento por DM; existe 
Tabla 2. Características generales de los pacientes con diagnóstico de tuberculosis con o sin la comorbilidad de diabetes mellitus.

\begin{tabular}{|c|c|c|c|}
\hline Características & $\begin{array}{c}\text { TB } \sin \text { DM } \\
(\mathbf{n}=52357)\end{array}$ & $\begin{array}{c}\text { TB con DM } \\
(\mathrm{n}=6577)\end{array}$ & Valor de $\mathbf{p}$ \\
\hline Hombre (\%) & $31904(62,3)$ & $3932(60,2)$ & 0,001 \\
\hline Mediana de la edad (RIC) & $33(24-51)$ & $53(41-63)$ & $<0,001$ \\
\hline \multicolumn{4}{|l|}{ Grupo etarios $(n=67$ 773) $(\%)$} \\
\hline $18-24$ & $13483(26,3)$ & $396(6,1)$ & \\
\hline $25-34$ & $13274(26,0$ & $604(9,3)$ & \\
\hline $35-44$ & $8055(15,8)$ & $1003(15,4)$ & $<0,001$ \\
\hline $45-54$ & $5323(10,4)$ & $1526(23,4)$ & \\
\hline $55-64$ & $4200(8,2)$ & $1520(23,3)$ & \\
\hline$\geq 65$ & $6749(13,2)$ & $1478(22,6)$ & \\
\hline VIH (\%) & $3284(6,7)$ & $256(4,2)$ & $<0,001$ \\
\hline TB extrapulmonar (\%) & $11366(22,2)$ & $790(12,1)$ & $<0,001$ \\
\hline Tabaquismo (\%) & $3002(5,7)$ & $280(4,3)$ & $<0,001$ \\
\hline \multicolumn{4}{|l|}{ Condición de egreso (\%) } \\
\hline Curado & $33241(64,9)$ & $4500(68,9)$ & \\
\hline Tratamiento completo & $10886(21,3)$ & $1170(17,9)$ & \\
\hline Perdido en el tratamiento & $4165(8,1)$ & $368(5,6)$ & $<0,001$ \\
\hline Fallecido & $2775(5,4)$ & $467(7,2)$ & \\
\hline Fracaso al tratamiento & $140(0,3)$ & $24(0,4)$ & \\
\hline Reacción adversa a medicamentos (\%) & $582(1,2)$ & $81(1,2)$ & 0,297 \\
\hline
\end{tabular}

DM: diabetes mellitus; RIC: rango intercuartil; TB: tuberculosis; VIH: virus de inmunodeficiencia humana.

evidencia que indica que los pacientes con DM controlada tienen un tratamiento exitoso, similar que aquellos pacientes con TB que no tienen DM ${ }^{(25)}$; asimismo, existe evidencia de que el uso de metformina podría estar asociado a un mejor resultado al tratamiento contra la $\mathrm{TB}{ }^{(26,27)}$, incluso reduce el riesgo de recaídas de TB luego de tres años de haber terminado el tratamiento ${ }^{(28)}$.

Uno de los problemas encontrados en este estudio fue la falta de datos relacionados con la DM, que no estaban incluidos en el SIGTB, como el tipo de tratamiento de DM, resultados de glucosa, hemoglobina glicosilada, perfil lipídico, adherencia al tratamiento para DM, índice de masa corporal, entre otras variables. Como no se toman como información importante para la evaluación del paciente con $\mathrm{TB}$, se tiende a ver las enfermedades como «silos» y eso no permite una adecuada evaluación integral, además de no poder aislar adecuadamente el efecto real de la DM en TB debido a variables de confusión que no fueron medidas ${ }^{(13)}$. Sin embargo, en las nuevas versiones del SIGTB se están incluyendo varias de estas variables, lo que permitirá una mejor vigilancia de la comorbilidad TB-DM.

Asimismo, debido a que el SIGTB no registra las causas específicas de muerte, en pacientes con TB, no se sabe si la causa de muerte ha sido por TB o por una causa cardiovas- cular o metabólica. Además, existe una falta de registro de alrededor del 14\% del estado de DM en la base (algunas regiones más que otras) que podrían subestimar la asociación encontrada. En el caso de la DM es importante tener una adecuada evaluación del diagnóstico, ya que es común en pacientes con TB (como en otras infecciones) un estado de hiperglicemia transitoria. Un estudio en Tanzania encontró que la hiperglicemia se redujo luego del tratamiento para $\mathrm{TB}$, pero a pesar de eso se encontró asociación entre la hiperglicemia al inicio del tratamiento con resultados adversos al tratamiento ${ }^{(29)}$. Debido a esto, la evaluación de la glicemia en pacientes con DM e incluso con hiperglicemia debe ser seguida durante el tratamiento de la TB para confirmar si es un caso de hiperglicemia transitoria. Finalmente, no se registra regularmente el tratamiento de la DM en la ficha de tratamiento de TB, ya que no existe mucha evidencia sobre el mejor tratamiento en estos casos. Los expertos sugieren que el uso de insulina es la mejor opción porque permite un manejo adecuado de la glucosa, pero implica una mayor participación del personal de salud para entrenar al paciente y a su entorno. Por otro lado, la metformina ha mostrado, en algunos estudios, actividad en contra del Mycobacterium tuberculosis ${ }^{(26,27,30)}$, pero aún no hay ensayos clínicos aleatoriza- 
Tabla 3. Modelo de regresión de Poisson para la asociación entre la diabetes mellitus y mortalidad en pacientes con tuberculosis $(n=55322)$.

\begin{tabular}{|c|c|c|c|c|}
\hline \multirow{2}{*}{ Factores } & \multicolumn{2}{|c|}{ Modelo crudo } & \multicolumn{2}{|c|}{ Modelo ajustado $^{\text {a }}$} \\
\hline & RR (IC 95\%) & Valor de p & RR (IC 95\%) & Valor de p \\
\hline \multicolumn{5}{|l|}{ DM } \\
\hline No & 1 (Ref.) & $<0,001$ & 1 (Ref.) & \\
\hline Sí & $1,32(1,20-1,50)$ & & $0,9(0,8-1,0)$ & 0,185 \\
\hline \multicolumn{5}{|l|}{ VIH } \\
\hline No & 1 (Ref.) & & 1 (Ref.) & \\
\hline Sí & $5,48(5,08-5,91)$ & $<0,001$ & $6,8(6,2-7,4)$ & $<0,001$ \\
\hline \multicolumn{5}{|l|}{ Sexo } \\
\hline Mujer & 1 (Ref.) & & 1 (Ref.) & \\
\hline Hombre & $1,31(1,23-1,41)$ & $<0,001$ & $1,3(1,2-1,4)$ & $<0,001$ \\
\hline \multicolumn{5}{|c|}{ Grupo etario (años) } \\
\hline $18-24$ & 1 (Ref.) & & 1 (Ref.) & \\
\hline $25-34$ & $2,1(1,8-2,5)$ & $<0,001$ & $1,7(1,4-2,0)$ & $<0,001$ \\
\hline $35-44$ & $3,2(2,7-3,7)$ & $<0,001$ & $2,3(2,0-2,8)$ & $<0,001$ \\
\hline $45-54$ & $4,4(3,8-5,2)$ & $<0,001$ & $3,7(3,1-4,4$ & $<0,001$ \\
\hline $55-64$ & $6,1(5,2-7,1)$ & $<0,001$ & $6,1(5,2-7,2)$ & $<0,001$ \\
\hline$\geq 65$ & $11,8(10,2-13,5)$ & $<0,001$ & $11,4(9,8-13,3)$ & $<0,001$ \\
\hline \multicolumn{5}{|c|}{ TB extrapulmonar } \\
\hline No & 1 (Ref.) & & 1 (Ref.) & \\
\hline Sí & $1,6(1,4-1,7)$ & $<0,001$ & $1,5(1,4-1,7)$ & $<0,001$ \\
\hline \multicolumn{5}{|c|}{ Antecedente de TB } \\
\hline No & 1 (Ref.) & 0,278 & 1 (Ref.) & 0,331 \\
\hline Sí & $1,1(1,0-1,2)$ & & $1,1(0,9-1,2)$ & \\
\hline
\end{tabular}

DM: diabetes mellitus; TB: tuberculosis; VIH: virus de inmunodeficiencia humana; RR: riesgo relativo.

a Ajustado por VIH, sexo y edad.

dos que confirmen este efecto, además de presentar eventos adversos como la acidosis láctica, nauseas o vómitos que pueden afectar la adherencia al tratamiento de la TB.

La fortaleza de este estudio es haber evaluado a todos los pacientes registrados en el Perú durante el 2016-2018, con resultados del tratamiento en casi todos los pacientes (>99\%), por lo que es representativo de ese periodo. La plataforma de SIGTB permite una mejor evaluación y seguimiento de esta comorbilidad, ya que admite realizar actualizaciones de datos importantes como la glucosa o el índice de masa corporal. Asimismo, permite identificar las zonas en el país donde aún no se llega al 100\% de tamizaje de DM (algo casi logrado en VIH), para poder reforzar el sistema de salud y lograr el objetivo de identificación de los pacientes con esta comorbilidad.

\section{REFERENCIAS BIBLIOGRÁFICAS}

1. International Diabetes Federation. IDF Diabetes atlas. Eighth edition. 2017. Disponible en: https://diabetesatlas.org/upload/resources/ previous/files/8/IDF_DA_8e-EN-final.pdf

2. World Health Organization. Global tuberculosis report 2020: executive summary. Disponible en: https://apps.who.int/iris/bitstream/ handle/10665/337538/9789240016095-eng.pdf
En conclusión, la DM ha sido identificada como la comorbilidad más común en pacientes con TB en el Perú. Se necesitan estudios que evalúen la cascada de cuidado en pacientes con TB y DM para evaluar las barreras y dificultades que presentan estos pacientes, así como para plantear un enfoque de cuidado de multimorbilidad dentro de la atención centrada en la persona.

Contribuciones de los autores: $\mathrm{CU}-\mathrm{G}, \mathrm{MC}, \mathrm{EH}, \mathrm{HH}$ y JR concibieron y diseñaron el artículo, e interpretaron los resultados. CU-G analizó los datos y elaboró la primera versión del artículo. Todos los autores revisaron y aprobaron la versión final del artículo.

Financiamiento: Autofinanciado.

Conflictos de interés: Los autores declaran no tener conflictos de interés.
3. Noubiap JJ, Nansseu JR, Nyaga UF, Nkeck JR, Endomba FT, Kaze AD, et al. Global prevalence of diabetes in active tuberculosis: a systematic review and meta-analysis of data from 2.3 million patients with tuberculosis. Lancet Glob Health. 2019;7(4):e448-e60. doi: 10.1016/s2214-109x(18)30487-x.

4. Odone A, Houben RM, White RG, Lönnroth K. The effect of diabetes and undernutrition trends on reaching 2035 global tuberculosis targets. 
Lancet Diabetes Endocrinol. 2014;2(9):754-64. doi: 10.1016/s22138587(14)70164-0.

5. Huangfu P, Ugarte-Gil C, Golub J, Pearson F, Critchley J. The effects of diabetes on tuberculosis treatment outcomes: an updated systematic review and meta-analysis. Int J Tuberc Lung Dis. 2019;23(7):783-96. doi: 10.5588/ijtld.18.0433.

6. Al-Rifai RH, Pearson F, Critchley JA, Abu-Raddad LJ. Association between diabetes mellitus and active tuberculosis: A systematic review and meta-analysis. PLoS One. 2017;12(11):e0187967. doi: 10.1371/journal. pone.0187967.

7. Pearson F, Huangfu P, McNally R, Pearce M, Unwin N, Critchley JA. Tuberculosis and diabetes: bidirectional association in a UK primary care data set. J Epidemiol Community Health. 2019;73(2):142-7. doi: 10.1136/jech-2018-211231.

8. Ranzani OT, Rodrigues LC, Bombarda S, Minto CM, Waldman EA, Carvalho CRR. Long-term survival and cause-specific mortality of patients newly diagnosed with tuberculosis in São Paulo state, Brazil, 2010-15: a population-based, longitudinal study. Lancet Infect Dis. 2020;20(1):12332. doi: 10.1016/s1473-3099(19)30518-3.

9. Ronacher K, van Crevel R, Critchley JA, Bremer AA, Schlesinger LS, Kapur A, et al. Defining a Research Agenda to Address the Converging Epidemics of Tuberculosis and Diabetes: Part 2: Underlying Biologic Mechanisms. Chest. 2017;152(1):174-80. doi: 10.1016/j.chest.2017.02.032.

10. Viney K, Mills T, Harley D. Tuberculosis and diabetes mellitus: a dose-response relationship between the odds of tuberculosis and HbAlc. Int J Tuberc Lung Dis. 2019;23(10):1055-9. doi: 10.5588/ijtld.18.0657.

11. Dirección de Prevención y Control de Tuberculosis. Sistema de Información Gerencial de Tuberculosis Data. Lima: MINSA; 2020. Disponible en: https://appsalud.minsa.gob.pe/sigtbdata/wflogin.aspx.

12. Ministerio de Salud. Norma técnica de salud para la atención integral de las personas afectadas por tuberculosis. Lima: MINSA; 2013.

13. Huangfu P, Pearson F, Ugarte-Gil C, Critchley J. Diabetes and poor tuberculosis treatment outcomes: issues and implications in data interpretation and analysis. Int J Tuberc Lung Dis. 2017;21(12):1214-9. doi: 10.5588/ ijtld.17.0211.

14. Jacob S, George LS, Joy A, Mathew MM, Vijayakumar K, Kumar A, et al. Prevalence of diabetes mellitus and HIV/AIDS among tuberculosis patients in Kerala. J Family Med Prim Care. 2020;9(12):6209-12. DOI: 10.4103/jfmpc.jfmpc_1583_20.

15. Martins-Melo FR, Bezerra JMT, Barbosa DS, Carneiro M, Andrade KB, Ribeiro ALP, et al. The burden of tuberculosis and attributable risk factors in Brazil, 1990-2017: results from the Global Burden of Disease Study 2017. Popul Health Metr. 2020;18(Suppl 1):10. doi: 10.1186/ s12963-020-00203-6.

16. Menon S, Rossi R, Dusabimana A, Zdraveska N, Bhattacharyya S, Francis J. The epidemiology of tuberculosis-associated hyperglycemia in individuals newly screened for type 2 diabetes mellitus: systematic review and meta-analysis. BMC Infect Dis. 2020;20(1):937. doi: 10.1186/s12879-020-05512-7.

17. Riza AL, Pearson F, Ugarte-Gil C, Alisjahbana B, van de Vijver S, Panduru $\mathrm{NM}$, et al. Clinical management of concurrent diabetes and tuberculosis and the implications for patient services. Lancet Diabetes Endocrinol. 2014;2(9):740-53. doi: 10.1016/s2213-8587(14)70110-x.
18. Mave V, Gaikwad S, Barthwal M, Chandanwale A, Lokhande R, Kadam $\mathrm{D}$, et al. Diabetes Mellitus and Tuberculosis Treatment Outcomes in Pune, India. Open Forum Infect Dis. 2021;8(4):ofab097. doi: 10.1093/ ofid/ofab097.

19. Ugarte-Gil C, Alisjahbana B, Ronacher K, Riza AL, Koesoemadinata RC, Malherbe ST, et al. Diabetes Mellitus Among Pulmonary Tuberculosis Patients From 4 Tuberculosis-endemic Countries: The TANDEM Study. Clin Infect Dis. 2020;70(5):780-8. doi: 10.1093/cid/ciz284.

20. Calderon RI, Arriaga MB, Lopez K, Barreda NN, Sanabria OM, Fróes Neto $\mathrm{JF}$, et al. High prevalence and heterogeneity of Dysglycemia in patients with tuberculosis from Peru: a prospective cohort study. BMC Infect Dis. 2019;19(1):799. doi: 10.1186/s12879-019-4416-2.

21. Alebel A, Wondemagegn AT, Tesema C, Kibret GD, Wagnew F, Petrucka $\mathrm{P}$, et al. Prevalence of diabetes mellitus among tuberculosis patients in Sub-Saharan Africa: a systematic review and meta-analysis of observational studies. BMC Infect Dis. 2019;19(1):254. doi: 10.1186/ s12879-019-3892-8.

22. Liu Q, Lu P, Martinez L, Peng H, Zhu T, Zhu L, et al. Undiagnosed diabetes mellitus and tuberculosis infection: A population-based, observational study from eastern China. Diabetes Metab Res Rev. 2020;36(3):e3227. doi: 10.1002/dmrr.3227.

23. Zheng Y, Ley SH, Hu FB. Global aetiology and epidemiology of type 2 diabetes mellitus and its complications. Nat Rev Endocrinol. 2018;14(2):8898. doi: 10.1038/nrendo.2017.151.

24. Huaman MA, Ticona E, Miranda G, Kryscio RJ, Mugruza R, Aranda E, et al. The Relationship Between Latent Tuberculosis Infection and Acute Myocardial Infarction. Clin Infect Dis. 2018;66(6):886-92. doi: 10.1093/ cid/cix910.

25. Critchley JA, Restrepo BI, Ronacher K, Kapur A, Bremer AA, Schlesinger LS, et al. Defining a Research Agenda to Address the Converging Epidemics of Tuberculosis and Diabetes: Part 1: Epidemiology and Clinical Management. Chest. 2017;152(1):165-73. doi: 10.1016/j.chest.2017.04.155.

26. Singhal A, Jie L, Kumar P, Hong GS, Leow MK, Paleja B, et al. Metformin as adjunct antituberculosis therapy. Sci Transl Med. 2014;6(263):263ra159. doi: 10.1126/scitranslmed.3009885.

27. Yu X, Li L, Xia L, Feng X, Chen F, Cao S, et al. Impact of metformin on the risk and treatment outcomes of tuberculosis in diabetics: a systematic review. BMC Infect Dis. 2019;19(1):859. doi: 10.1186/s12879-019-4548-4.

28. Ma Y, Pang Y, Shu W, Liu YH, Ge QP, Du J, et al. Metformin reduces the relapse rate of tuberculosis patients with diabetes mellitus: experiences from 3-year follow-up. Eur J Clin Microbiol Infect Dis. 2018;37(7):125963. doi: 10.1007/s10096-018-3242-6.

29. Boillat-Blanco N, Ramaiya KL, Mganga M, Minja LT, Bovet P, Schindler C, et al. Transient Hyperglycemia in Patients With Tuberculosis in Tanzania: Implications for Diabetes Screening Algorithms. J Infect Dis. 2016;213(7):1163-72. doi: 10.1093/infdis/jiv568.

30. Rodriguez-Carlos A, Valdez-Miramontes C, Marin-Luevano P, González-Curiel I, Enciso-Moreno JA, Rivas-Santiago B. Metformin promotes Mycobacterium tuberculosis killing and increases the production of human $\beta$-defensins in lung epithelial cells and macrophages. Microbes Infect. 2020;22(3):111-8. doi: 10.1016/j.micinf.2019.10.002. 